\title{
Unidad de Energía Renovable del Centro de Investigación Científica de Yucatán
}

\author{
Juan Carlos Chavarría Hernández, ${ }^{*}$ Luis Carlos Ordóñez López, ** \\ Beatriz Escobar Morales, *** Daniela Esperanza Pacheco Catalán****
}

RESUMEN: En esta contribución se describen las actividades de investigación y desarrollo tecnológico que se realizan en áreas relacionadas con la catálisis, así como con nanociencias y nanotecnología (NyN) en la Unidad de Energía Renovable (UER) del Centro de Investigación Científica de Yucatán (CICY). Se hace una breve reseña de los orígenes de la UER del CICY, así como del número de investigadores y técnicos académicos que desarrollan temas de investigación relacionados con la catálisis y nanotecnología. Asimismo, se describe de manera resumida la infraestructura con la que cuenta actualmente la UER del CICY disponible para la ejecución de proyectos relacionados con estos temas de investigación. Se menciona también un listado de instituciones de investigación y educación superior con las cuales la UER del CICY tiene colaboraciones a diferentes niveles de formalidad para la realización de los proyectos de investigación relacionados con catálisis y nanotecnología. Por otra parte, se hace mención de los programas de maestría y doctorado impartidos en la UER del CICY a través de los cuales se forman recursos humanos especializados en áreas de energía renovable, indicando el número de estudiantes de posgrado, asi como de licenciatura que la UER del CICY ha formado en temas relacionados con catálisis y nanotecnología. Finalmente, se describen brevemente algunas áreas de oportunidad y perspectivas de crecimiento en los temas referidos en el corto y mediano plazos.

PALABRAS CLAVE: UER-CICY, líneas de investigación, energía renovable, catalizadores heterogéneos, electrocatalizadores.

ABSTRACT: This contribution describes the research and technological development activities carried out in areas related to catalysis, as well as nanosciences and nanotechnology (NyN) at the Renewable Energy Unit (UER) of the Center for Scientific Research of Yucatan (CICY). A brief overview of the origins of the UER is made, and the number of researchers and academic technicians who develop research topics related to catalysis and nanotechnology is mentioned. The infrastructure currently available at the UER for the execution of projects related to these research topics is also described in a summarized way. A list of research and higher education institutions with which UER collaborates in different levels of formality for the realization of research projects related to catalysis and nanotechnology is also given. On the other hand, mention is made of the master's and doctoral programs taught at the UER, through which specialized human resources are trained in the areas of renewable energies, as well as the number of graduate and undergraduate students who have developed their thesis projects at the UER on

Recibido: 24 de enero de 2017. Aceptado: 3 de mayo de 2017.

* CICY-Unidad de Energía Renovable (UER), Parque Científico y Tecnológico de Yucatán. Carretera Sierra Papacal-Chuburna Puerto, km 5. Sierra Papacal, C.P. 97302, Mérida, Yucatán, Tel.: (999)930 0760, ext. 1206. Correspondencia: (jc.ch@cicy.mx).

** CICY-UER. Tel.: (999)930 0760, ext. 1205. Correspondencia: (lcol@cicy.mx).

*** CICY-UER. Tel.: (999)930 0760, ext. 1203. Correspondencia: (beatriz.escobar@cicy.mx).

**** CICY-UER. Tel.: (999)930 0760, ext. 1202. Correspondencia: (dpacheco@cicy.mx). 
Mundo Nano | CATÁLISIS EN MÉxICo | www.mundonano.unam.mx

10(18), enero-junio 2017 | DOI: 10.22201/ceiich.24485691e.2017.18.60109

issues related to catalysis and nanotechnology. Finally, some areas of opportunity and growth prospects in the aforementioned topics in the short and medium terms are briefly described. KEYWORDS: UER-CICY, research lines, renewable energy, heterogeneous catalysts, electrocatalysts.

\section{Introducción}

La Unidad de Energía Renovable (UER) del Centro de Investigación Científica de Yucatán (CICY), cuya fachada principal se muestra en la figura 1, es una de las seis unidades académicas (Bioquímica y Biología Molecular de Plantas, Biotecnología, Ciencias del Agua, Materiales, Recursos Renovables y Energía Renovable) de este Centro, el cual pertenece al Sistema de Centros Públicos de Investigación del Consejo Nacional de Ciencia y Tecnología (CONACyT). La UER se creó en 2010 como producto de la evolución de una parte del grupo de trabajo de la Unidad de Materiales enfocada al desarrollo de aplicaciones en temas de energía, así como del Programa Institucional de Bioenergía creado en el CICY en 2006. Su misión es fomentar las fuentes de energía renovable a través de la investigación científica, el desarrollo tecnológico y la formación de recursos humanos de alto nivel, enfocándose en temas de bioenergía, tecnología del hidrógeno y sistemas híbridos de energía. Actualmente, la UER cuenta con diez investigadores, de los cuales cuatro realizan investigación o desarrollo tecnológico en áreas relacionadas con catálisis y nanotecnología, apoyados por cuatro técnicos académicos. En la UER se imparten programas de maestría y doctorado inscritos en el Padrón Nacional de Posgrados de Calidad (PNPC) del CONACyT, siendo la población actual de alumnos de posgrado de aproximadamente 60 estudiantes. En el 2013, la UER movió su sede del campus Mérida del CICY al Parque Científico y Tecnológico de Yucatán, localizado en la carretera Sierra Papacal-Puerto de Chuburná. Dicho parque es un polo de desarrollo científico y tecnológico de la región, que reúne a varias instituciones de alto nivel como son: CINVESTAV, CIATEJ, CIMAT, CIESAS, UNAM, UADY, CICY, así como a empresas y dependencias científicas como la Secretaría de Investigación, Innovación y Educación Superior del Estado de Yucatán y el conACyT, entre otras.

\section{Líneas de investigación, desarrollo e innovación relacionadas con la catálisis}

En la UER se desarrollan tres líneas de generación y aplicación del conocimiento: bioenergía, tecnología del hidrógeno y sistemas híbridos de energía. Las dos primeras se crearon en 2010 y la última en 2016. En la línea de bioenergía se sintetizan y evalúan catalizadores para estudiar la transformación de biomasa en combustibles líquidos renovables, principalmente bioturbosina, diésel renovable, biogasolina y biodiesel. Entre los materiales empleados se encuentran zeolitas tales como la ZSM-22 y la ZSM-5; materiales 


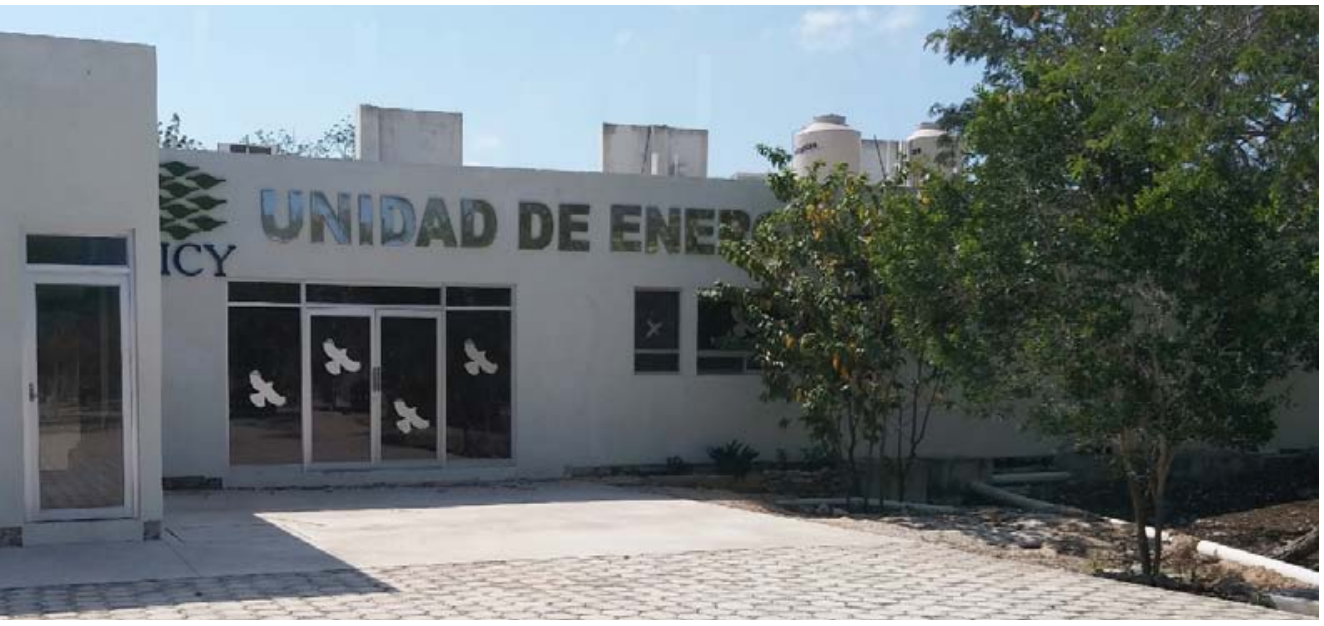

FIGURA 1. Entrada al edificio principal del edificio de la Unidad de Energía Renovable del Centro de Investigación Científica de Yucatán.

mesoporosos como son la SBA-15 y la MCM-41. Los prototipos catalíticos son caracterizados por diferentes técnicas fisicoquímicas (XRD, SEM-EDX, FTIR, fisisorción de $\mathrm{N}_{2}$, etc.), posteriormente su actividad es evaluada en procesos de obtención de biocombustibles que incluyen la hidrodesoxigenación (HDO) o hidrotratamiento (HT), y un proceso catalítico de tres etapas de reacción consecutivas conocido como ATJ (alcohol to jet). Como materia prima para el proceso de HDO se utilizan triglicéridos y otros lípidos presentes en aceites vegetales (jatropha, higuerilla), grasas animales (pollo), aceites comestibles usados y aceite de microalgas. También se estudia una modalidad de desoxigenación catalítica (DO) a baja presión y bajo consumo de hidrógeno. Para el proceso ATJ se emplea bioetanol como materia prima. La caracterización de los productos de reacción se realiza mediante técnicas de GC-MS, FTIR y análisis elemental CHONS principalmente.

En la línea de tecnología del hidrógeno se trabaja con distintos materiales a escala nanométrica cuyo fin es utilizarlos como fases activas o soportes de electrocatalizadores para las reacciones de electrodo en celdas de combustible de hidrógeno de membrana de intercambio protónico (PEM-FC), celdas de combustible de alcohol directo (DAFC), o bien, en dispositivos electroquímicos de almacenamiento de energía como son capacitores electroquímicos (supercapacitores) o baterías. Las principales reacciones electroquímicas que se estudian son: la electroxidación de alcoholes de bajo peso molecular como metanol o etanol, la reducción electroquímica de oxígeno o de $\mathrm{CO}_{2}$, la evolución de oxígeno; así como también se estudian los fenómenos de almacenamiento de carga electrostática o por procesos pseudocapacitivos como reacciones redox, procesos de adsorción o de intercalación, los cuales pueden estudiarse por medio de los procesos cíclicos de carga y descarga. 
Para la obtención de materiales para las diferentes aplicaciones, se emplean como fases activas metálicas nanopartículas de Pt, Pd, Ni promovidas con $\mathrm{Mo}, \mathrm{Ru}, \mathrm{Sn}$, etc., y como soportes se emplean materiales de carbono como negro de humo, nanotubos de carbono, óxido de grafeno, polímeros conductores como polipirrol, polianilina, o bien, distintos materiales compuestos. También se estudia la incorporación de nitrógeno o azufre como agentes dopantes de los soportes catalíticos. Los materiales son sintetizados a través de distintos procesos entre ellos: métodos coloidales, por medio del uso de plantillas, el uso de diferentes reductores químicos de sales precursoras, etc. La evaluación física de estos materiales se realiza por medio de la difracción de rayos $\mathrm{X}$ (DRX), espectroscopía fotoelectrónica de rayos $\mathrm{X}$ (XPS), microscopías electrónicas de barrido o de transmisión. Además, se emplean las técnicas espectroscópicas de infrarrojo (FTIR), RAMAN, y análisis elemental (CHONS). Otra técnica que también se emplea es la microscopía de fuerza atómica (AFM), la cual permite evaluar distintas propiedades de la superficie de los electrodos. Finalmente, las determinaciones de la actividad catalítica se realizan por medio de técnicas electroquímicas de corriente directa y espectroscopía de impedancia electroquímica. Posteriormente, los materiales obtenidos se prueban en prototipos de celdas de combustible o de supercapacitores desarrollados en la misma UER.

Además del desarrollo de catalizadores inorgánicos, poliméricos y organometálicos, se desarrollan membranas; se optimiza la fabricación de los ensambles electrodo-membrana-electrodos (EME), se simulan y fabrican diferentes prototipos a pequeña escala de platos bipolares, celdas PEM y celdas reversibles (unificadas).

\section{Colaboraciones y proyectos}

La Unidad de Energía Renovable del cicy colabora con diferente grado de acercamiento con distintas instituciones de educación superior (IES) y centros públicos de investigación (CPI) de la península de Yucatán y otras regiones del país para la elaboración de proyectos de investigación y desarrollo tecnológico, así como la codirección de tesis de licenciatura, maestría y doctorado. Entre estas instituciones se encuentran:

- Universidad Autónoma del Carmen (UNACAR).

- Universidad de Quintana Roo (UQROo).

- Universidad Autónoma de Yucatán (UADY).

- Facultad de Química de la UNAM.

- Universidad Politécnica de Tlaxcala.

- Universidad Juárez Autónoma de Tabasco (UJAT).

- Universidad de Guanajuato.

- Universidad Autónoma del Estado de Hidalgo.

- Universidad de Querétaro. 
- Instituto Tecnológico de Chetumal.

- Instituto Tecnológico de Mérida.

- Instituto Tecnológico de Ciudad Madero.

- Instituto Tecnológico de Cancún.

- Instituto Tecnológico de Tuxtla Gutiérrez.

- Cinvestav, unidad Mérida y unidad Saltillo.

- Instituto de Energías Renovables de la UNAM.

- Instituto Mexicano del Petróleo, sede Ciudad de México, entre otros.

La colaboración en el tema de biocombustibles se ha realizado principalmente para el desarrollo y evaluación de actividad de catalizadores para los procesos de hidrodesoxigenación de aceites en la obtención de hidrocarburos con potencial para emplearse como combustibles renovables: bioturbosina y diésel verde. En el tema de tecnología del hidrógeno se ha colaborado en la preparación de nanoestructuras de polímero intrínsecamente electroconductor, así como el estudio de su actividad electrocatalítica y de sus propiedades en sistemas electroquímicos de energía. Otro tema de colaboración es la electroxidación de etanol empleando catalizadores bimetálicos PtMe (Me = Mo, Sn y Ru) soportados ya sea en carbón, polipirrol o titania, así como el desarrollo de prototipos de respaldo de energía en casos de desastre natural. Uno de los proyectos emblemáticos que lideró la UER del CICY trabajando en colaboración con otras siete instituciones de la región es el proyecto Laboratorio de Energías Renovables del Sureste (LENERSE), creado para impulsar la implementación de las energías renovables en la región sureste del país, mediante el desarrollo de tecnologías, la investigación científica, la vinculación y la formación de recursos humanos (ver figura 2).

FIGURA 2. Edificio del Laboratorio de Energías Renovables del Sureste (LENERSE), ubicado a un costado del edificio de la UER.

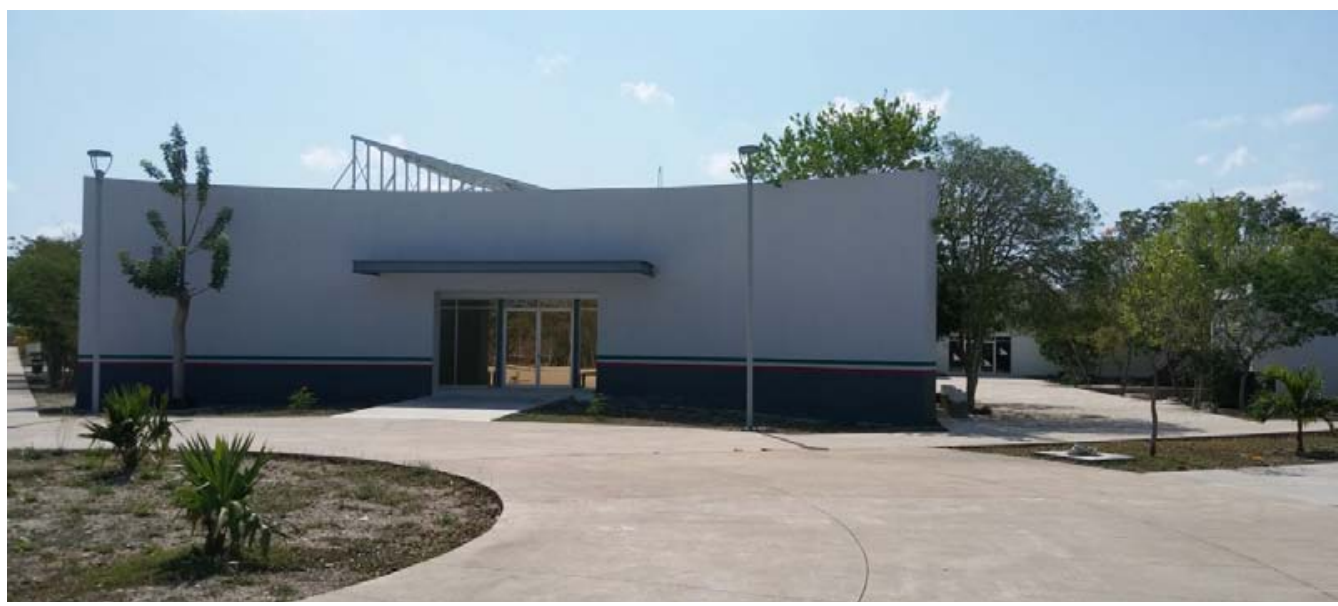


Este proyecto tendrá su continuación en el proyecto Consolidación del LENERSE, recientemente aprobado y que será financiado por el fondo de sustentabilidad energética SENER-CONACYT.

Por otra parte, los académicos de la UER que desarrollan proyectos relacionados con la catálisis tienen una participación activa en la Red Temática del Hidrógeno y en la Red Temática de Bioenergía, ambas del CONACyT, así como en el Clúster de Bioturbosina, uno de los cinco clústers (biocombustibles sólidos, bioalcoholes, biodiesel, biogás y bioturbosina) que conforman el Centro Mexicano de Innovación en Bioenergía (CEMIE-Bio), financiado por la Secretaría de Energía y el conACyT a través del Fondo de Sustentabilidad Energética. En el Clúster Bioturbosina, la UER del CICY participa con las siguientes instituciones, que incluyen a otros siete centros CONACYT:

- Centro de Investigaciones Biológicas del Noroeste (CIBNOR).

- Instituto Potosino de Investigación Científica y Tecnológica (IPICyT).

- Centro de Tecnología Avanzada (CIATeQ).

- Centro de Investigación en Alimentos y Desarrollo (CIAD).

- Centro de Investigación en Química Aplicada (CIQA).

- Centro de Investigación y Asistencia en Tecnología y Diseño del Estado de Jalisco (CIATEJ).

- Centro de Investigación y Desarrollo Tecnológico en Electroquímica (CIDETEQ).

- Instituto Mexicano del Petróleo (IMP).

- Instituto MASDAR, de los Emiratos Árabes Unidos.

Además, en este proyecto se firmó un convenio de entendimiento entre los participantes del Clúster, Boeing, Aeroméxico y Aeropuertos y Servicios Auxiliares (ASA).

En lo que respecta a la colaboración internacional, se han establecido vínculos para desarrollar conjuntamente temas relacionados con la catálisis y/o NyN con las siguientes instituciones:

- Universidad de Purdue, en Estados Unidos.

- Institute for Advanced Energy Technologies "Nicola Giordano" (CNRITAE).

- Instituto de Tecnología de Polímeros del csic, en Madrid España.

\section{Infraestructura}

La Unidad de Energía Renovable del cicy cuenta con los laboratorios de tecnología del hidrógeno, biocombustibles, biorreactores, síntesis, sistemas híbridos de energía, caracterización de materiales, así como con una planta piloto-taller y un cuarto de cultivo, sumando un área de 1,200 $\mathrm{m}^{2}$. Los edificios 
de la UER tienen espacios y servicios para poder realizar adecuadamente actividades de investigación, desarrollo tecnológico y formación de recursos humanos de alto nivel. Los principales equipos con que cuenta actualmente la UER son:

- Microscopio de fuerza atómica.

- Cromatógrafo de gases.

- Dos cromatógrafos de gases acoplados a detectores de masas.

- Cromatógrafo de líquidos de alta resolución.

- Ultra-congelador.

- Espectrofotómetros UV-vis, FTIR y RAMAN.

- Analizador elemental CHONS.

- Difractómetro de rayos X D2 Phaser.

- Reactor de lecho fijo para estudios de actividad catalítica.

- Hornos para calcinación, uno horizontal con tres zonas de calentamiento hasta $1000{ }^{\circ} \mathrm{C}$ y dos verticales hasta $600^{\circ} \mathrm{C}$.

- Potenciostatos-galvanostatos.

- Sistema de electrodo de disco y anillo rotatorio.

- Estaciones de evaluación de celdas de combustibles.

- Equipo de análisis textural (BET).

- Mesa óptica.

- Cámaras de electroforesis para gel de agarosa y de poliacrilamida.

- Termociclador.

- Fotodocumentador.

- Evaporador rotatorio.

- Cortadores con control numérico.

También cuenta con equipos convencionales como campanas de extracción y de flujo laminar, incubadoras, estufa de secado, refrigeradores, parrillas de calentamiento y agitación, balanzas, potenciómetros, sistema de purificación de agua, bombas de vacío y otros equipos auxiliares en el quehacer diario del personal y estudiantes. En las figuras 3 a 5 se muestran algunos de los equipos mencionados.
FIGURA 3 Reactor de lecho fijo modelo, microactivity reference, para evaluación de actividad catalítica y equipo de fisisorción de nitrógeno.
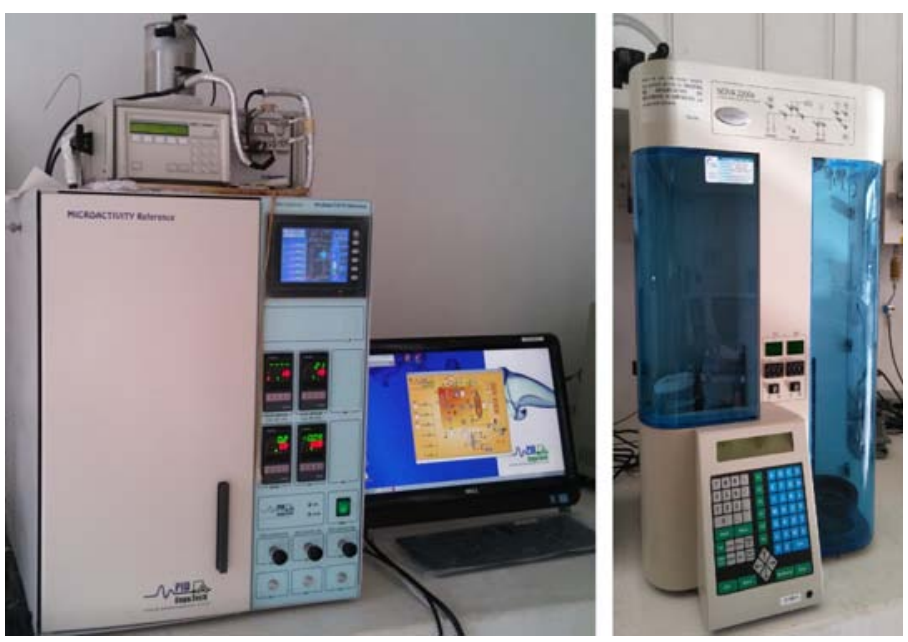
Mundo Nano | CATÁLISIS EN MÉXICo | www.mundonano.unam.mx

10(18), enero-junio 2017 | DOI: 10.22201/ceiich.24485691e.2017.18.60109
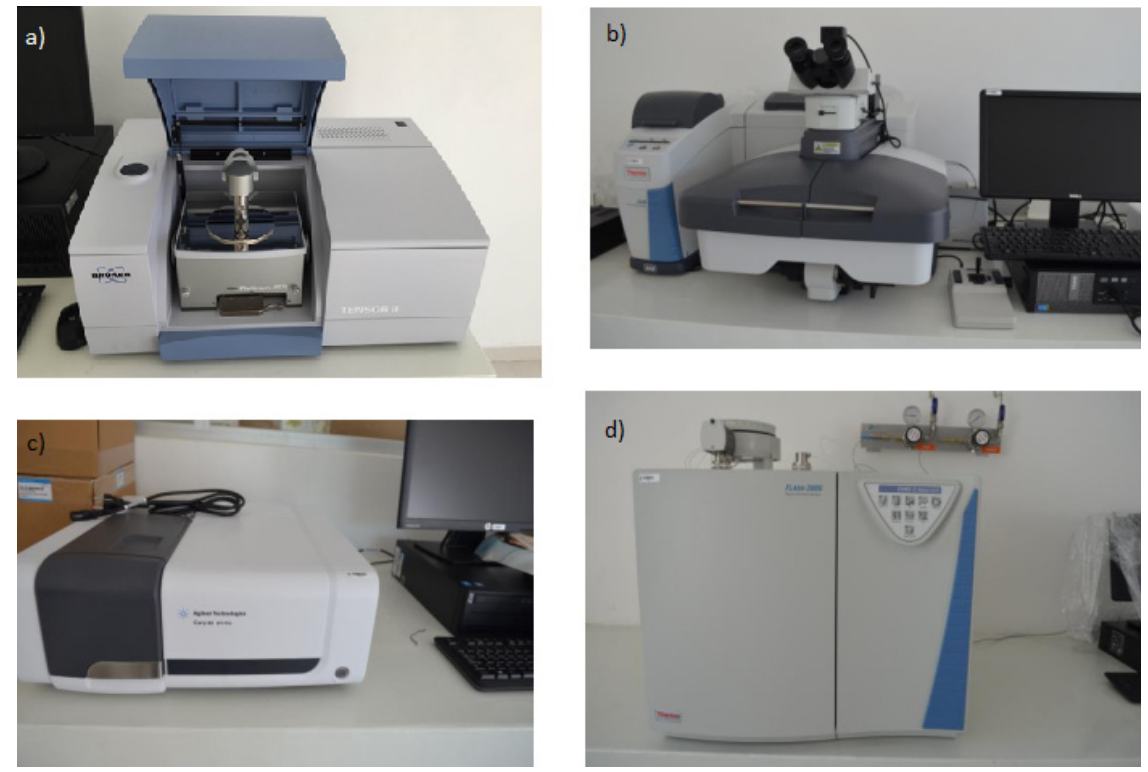

FIGURA 4. Equipos del Laboratorio de Caracterización de Materiales: a) FTIR; b) Raman; c) UV-vis, y, d) analizador elemental CHONS.

FIGURA 5. a) Laboratorio de Electrocatálisis; b) microscopio de fuerza atómica (AFM); c) estaciones de celdas de combustible, y, d) electrodo de disco rotatorio y de anillo.
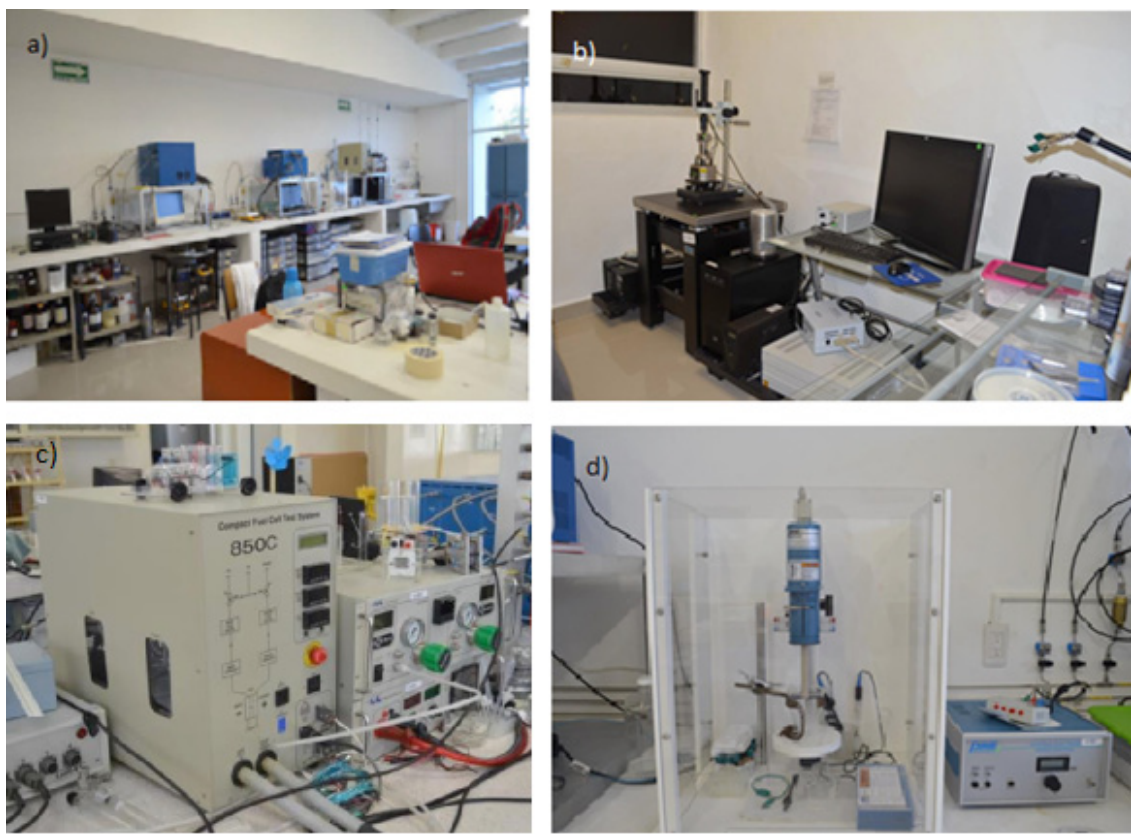


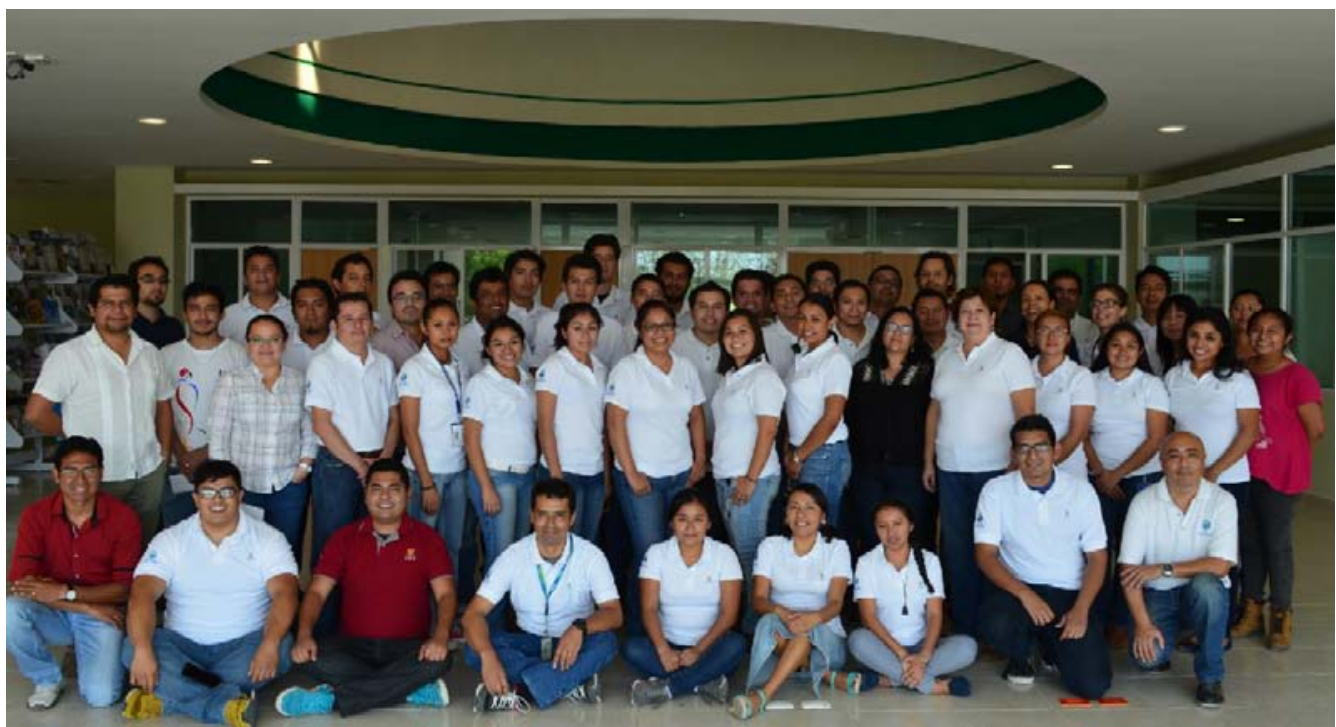

FIGURA 6. Académicos y estudiantes de la UER del CICY, 2015.

\section{Docencia y formación de recursos humanos}

Para la formación de recursos humanos, la UER cuenta con un programa de doctorado en ciencias y uno de maestría en ciencias en energía renovable, enfocados a la investigación y pertenecen al PNPC. El programa de maestría comenzó en 2008 y actualmente tiene el nivel de consolidado, mientras que el programa de doctorado inició en 2010 y está catalogado como un posgrado en desarrollo. Se atiende principalmente a egresados de la región sureste, si bien se cuenta con participación de estudiantes del centro y norte del país, así como con algunos estudiantes extranjeros. Los alumnos que ingresan son de las carreras de biología, física, química y de ingenierías afines. Adicionalmente, la UER de CICY atiende estudiantes de licenciatura para el desarrollo de servicio social, prácticas profesionales o realización de tesis. La formación de recursos humanos en temas de catálisis, NyN, incluye hasta ahora 2 tesis de doctorado, 12 de maestría y 11 de licenciatura concluidas. En la siguiente figura se muestra el personal científico y técnico de la UER, así como a los estudiantes de posgrado adscritos a esta Unidad para el año 2015.

\section{Principales logros en el área de la catálisis}

Se han desarrollado catalizadores efectivos para la desoxigenación de aceites y grasas y la obtención de hidrocarburos con buenas propiedades para emplearse como combustibles líquidos renovables, diésel verde y bioturbosina. Los materiales estudiados están considerados para llevar a cabo la desoxige- 
nación más isomerización más el rompimiento en una sola etapa de reacción en el procesamiento de lípidos. En este proceso se han empleado catalizadores metálicos soportados en materiales zeolíticos. Por otra parte se ha avanzado en el estudio de una modalidad de la desoxigenación que consiste en un menor consumo de hidrógeno y que permite de igual manera obtener hidrocarburos con alto valor para emplearse como combustible diésel. Para este proceso se han empleado principalmente catalizadores soportados en materiales mesoporosos como la SBA-15.

Asimismo, se ha avanzado en el desarrollo de electrocatalizadores nanométricos para la oxidación de alcoholes de bajo peso molecular en celdas de combustible. Se han obtenido buenos resultados con bajos contenidos de metales nobles $(\mathrm{Pt})$ al incorporar promotores como Ru, Mo, Sn. También se ha logrado obtener actividades catalíticas importantes en condiciones alcalinas al emplear fases activas basadas en Ni. Uno de los problemas para el desarrollo de catalizadores para celdas de combustible es el proceso de corrosión que sufren los soportes de carbón; como alternativa se han desarrollado soportes basados en polímeros intrínsecamente conductores con tamaños de partícula controlada.

También se tienen avances importantes en la obtención de materiales nanométricos para el almacenamiento electroquímico de carga para su aplicación en electrodos de supercapacitores. Se han establecido metodologías para la obtención y funcionalización de óxido de grafeno para evitar su reapilamiento. Y se ha obtenido grafeno por CVD, en no más de 5 capas, por lo que se continúa trabajando para lograr la obtención de la monocapa.

\section{Perspectivas sobre el estudio de la catálisis}

En el grupo de trabajo de la UER-CICY que trabaja en temas de catálisis y nanotecnología se vislumbran entre otras las siguientes áreas de oportunidad y perspectivas de crecimiento en el corto y mediano plazo:

Desarrollar catalizadores más eficientes para el proceso de obtención de combustibles líquidos renovables a partir de aceites y grasas, con los cuales sea posible modular de manera más adecuada el nivel de craqueo de forma tal que la pérdida de rendimiento líquido sea mínima, mientras se logran niveles de isomerización altos para permitir obtener combustibles con buenas propiedades de flujo en frío. Por otra parte, en el tema de catalizadores para el proceso ATJ a partir de bioetanol, se trabajará de manera especial en el desarrollo de catalizadores efectivos para la reacción de oligomerización de etileno, siendo ésta la reacción crítica para lograr la viabilidad técnica y económica del proceso.

En otro tema, se ha identificado que uno de los problemas que impiden que la comercialización de las celdas de combustible sea factible es el alto costo de los materiales catalíticos y la tolerancia que éstos tienen hacia la 
presencia de $\mathrm{CO}$ y otras especies intermediarias. En este sentido se pretende desarrollar materiales catalíticos libres de metales como Pt, Ru, Pd o Ir. Se busca trabajar en condiciones alcalinas, es decir, emplear como electrolitos, membranas de intercambio aniónico. Esto abre la posibilidad de emplear como fase activa metales económicos como el $\mathrm{Ni}$. De esta manera, se examina la posibilidad de incrementar la actividad del Ni a través de la modificación de su estructura, el dopaje o la deposición sobre soportes con propiedades interesantes.

Por otra parte, se continuará con el desarrollo de materiales nanoestructurados para aplicaciones en tecnología del hidrógeno y energías renovables, y su consecuente implementación en prototipos de generación de energía más amigables con el medio ambiente. Otro aspecto que es de interés del grupo a corto plazo se centra en la síntesis verde, caracterización y funcionalización de nanopartículas metálicas con aplicaciones en la generación y almacenamiento electroquímico de energía. Asimismo, se está trabajando para ampliar la variedad de partículas metálicas soportadas en materiales a base de grafeno con aplicación para las reacciones de reducción de oxígeno y de evolución de hidrógeno.

\section{Sitios de interés relacionados con la UER-CICY}

- 〈www.cicy.mx>

- <http://www.cicy.mx/unidad-de-energia-renovable/introduccion>

- <http://www.cicy.mx/posgrado-en-energia-renovable/inicio> 